\title{
EFFECTS OF QUANTUM CONFINEMENTS IN TIN SulPhide NANOCRYSTAls PROduCED BY WET-SOLUTION TECHNIQUE
}

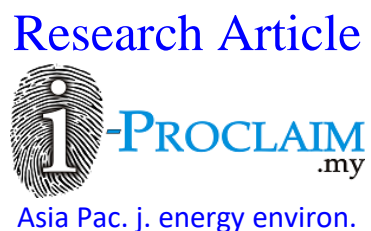

\section{Cliff Orori Mosiori}

Lecturer, Department of Mathematics and Physics, Technical University of Mombasa, Mombasa, KENYA

*Email for Correspondence: corori@tum.ac.ke

Abstract

In thin film nano-crystals studies, electron energy levels are known not continuous in the bulk thin films but are rather discrete(finite density of states) because of confinement of their electron wave functions to the physically dimensions of the particles. This phenomenon is called Quantum confinement and therefore nano-crystals are also referred to Quantum dots. The quantum confinement effect is mainly observed when the size of the particle involved is too small to be comparable to the wavelength of the electron. To understand this effect, this study broke the words confinement to mean to confine the motion of randomly moving electron to restrict its motion in specific energy levels (discreteness) and the term quantum to reflect the atomic realm of particles involved in this study. So as the size of a particle decrease to a nano scale, then the decrease in confining dimension causes the particle energy levels to be too discrete and at the same time widens up the band gap. As a result the ultimately effect is that the band gap energy increases. In this study, nanocrystalline tin sulphide $(\mathrm{SnS})$ powder was prepared using tin chloride $(\mathrm{SnCl} 2)$ as a tin ion $(\mathrm{Sn}+2)$ source and sodium sulfide (Na2S) as a sulfur (S-2) ion source using solution magneto DC sputtering technique. The as-synthesized thin film in form of nanoparticles were then qualitatively and quantitatively analyzed and characterized in terms of their morphological, structural and optical properties and found to have an orthorhombic structure whose direct band gap had blue shifted (1.74 $\mathrm{eV}$ ) and was confirmed using theoretical calculations of exciton energy based on the potential morphing method (PMM) in the Hartree Fock approximation.

Key words

Potential Morphing Method; Quantum Confinement effect; Hartree Fock Approximation

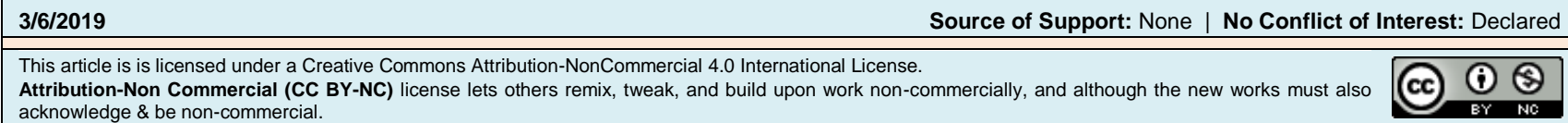

\section{INTRODUCTION}

The study of quantum confinement effect in thin films has created a great interest in the recent years because thin films exhibit different and unique optoelectronic properties from those of bulk thin films. Therefore, they are considered as a new class of materials that hold considerable attention for numerous applications in the field of optoelectronics. A lot of investigations has been done on the reduction in the size of particles to nanometer ranges and their related changes in the degree of confinement of charge carriers, which affect the electronic and optical properties of semiconductor materials (Poulopoulos et al., 2011). From theoretical point of view, as the radius of particle approaches the exciton Bohr radius of a given material, quantization of the energy bands become apparent and a blue shift in the exciton transition energy can be observed (Vidal et al., 2012). Tin sulfide has a very narrow band gap. It has low toxicity with a variety of optoelectronic applications that include as an absorber layer in solar cells, in holographic recording media and solar control devices (Goharshadi et al., 2009).

Experimental studies indicate that $\mathrm{SnS}$ exhibit both $\mathrm{p}$ and $\mathrm{n}$ type conduction depending on its preparation conditions and has both a direct optical gap located at $1.3 \mathrm{eV}$ and an indirect optical band gap located at $1.1 \mathrm{eV}$ (Goharshadi et al., 2009). This is based on the findings by spray pyrolysis of the water solution (Bashkirov et al., 2011), vacuum evaporation (Yue et al., (2009), chemical vapor deposition (Azizian-Kalandaragh and Khodayari, 2010), chemical bath deposition (Vidal et al., (2012), electro deposition and electrochemical deposition (Ning et al., 2010), chemical 
synthesis (Poulopoulos et al., 2011), microwave assisted synthesis (Mahendia et al., 2011), mild solution route (Azizian-Kalandaragh et al., 2009), modified solution dispersion method (Azizian-Kalandaragh and Khodayari, 2010), two gas process (Liu et al., 2010), solvothermal process (Gontard et al., 2011) among others. In this paper we report the preparation of SnS nanocrystals and its many advantages, such as easier composition control, low toxicity, better homogeneity, low processing temperature, easier fabrication of large numbers of nanoparticles, lower cost, and possibility of using high purity starting materials. We also report its morphological, optical, and structural properties.

\section{THEORY CONSIDERATIONS}

Quantum confinement is change of electronic and optical properties when a sampled material is of sufficiently small size - 10 nanometers or less. Specifically, the phenomenon results from electrons and holes being squeezed into a dimension that approaches a critical quantum measurement, called the exciton Bohr radius. This effect can be observed once the diameter of a material in question is of the same magnitude as the de Broglie wavelength of its electron wave function. This means that when materials involved are this small, their electronic properties, as well as their optical properties deviate substantially from those of its bulk nature. This implies that when a particle behaves as like it is free when it's confining dimension is large as compared to the wavelength of the particle. During this state, the band gap remains at its original energy due to it's continuous energy state. However, as the confining dimensions decreases and reaches a certain limit in nano-scale range, the energy spectrum becomes discrete and as a consequence, its band gap becomes size-dependent. The ultimately results is simply a blue shift in light emission and this is the effect that characterizes electrons and electron holes as being squeezed into a dimension that approaches critical quantum measurement, often called the exciton Bohr radius.

Using the effective mass approximation (Poulopoulos et al., 2011), the Hamiltonian for the electron hole system can be written as;

$$
H=-\frac{\hbar^{2}}{2 m_{e}^{*}} \nabla_{e}^{2}-\frac{\hbar^{2}}{2 m_{h}^{*}} \nabla_{h}^{2}+V_{0}^{e}\left(r_{e}\right)+V_{0}^{h}\left(r_{h}\right)-\frac{e^{2}}{\varepsilon} \frac{1}{r_{e h}}
$$

where $m_{e}^{*}\left(m_{h}^{*}\right)$ is the effective electron (hole) band mass, $\varepsilon$ is the effective dielectric constant, $r_{\mathrm{eh}}$ is the electron hole distance in three dimensions and $V_{0}^{e(h)}$ is the finite depth well confinement potential of electron (hole). Using the dielectric constant, $\varepsilon$ as developed by Mahendia et al. (2011); Azizian-Kalandaragh and Khodayari (2010) and which has the following form;

$$
\frac{1}{\varepsilon\left(R_{0}\right)}=\frac{1}{\varepsilon_{\infty}}-\left[\frac{1}{\varepsilon_{\infty}}-\frac{1}{\varepsilon_{0}}\right]\left[1-\frac{\exp \left(-R_{0} / \rho_{e}\right)+\exp \left(-R_{0} / \rho_{h}\right)}{2}\right]
$$

where $R_{0}$ is the mean distance between the electron and hole and approximately takes the values 0.69932R (Vidal et al., 2012) or $R$ (Azizian-Kalandaragh et al., 2009), where $R$ is the radius of the cluster and represents the half of the confining parameter which is the diameter of the nanocrystal. $\varepsilon_{0}$ and $\varepsilon_{\infty}$ are the static and optical dielectric constants respectively, and $\rho_{e, h}$ then it follows that;

$\rho_{e, h}=\left(\frac{\hbar}{2 m_{e, h}^{*} \omega_{L O}}\right)^{1 / 2}$

where $\omega_{L O}$ is the frequency of LO phonons. Thus, the height of the finite depth well confining potentials $V_{0}^{e(h)}$ for electrons and holes is independent of the nanostructured semiconductor material and therefore depends exclusively on the matrix energy band gap $E_{g}(M)$ by a simple linear relation of the form (Azizian-Kalandaragh and Khodayari, (2010);

$V_{0}=0.08 \cdot E_{g}(M)$ 
If we let that the confining potential has the same value for both electron and hole, then we can set for our thin film system to have;

$V_{0}^{e}\left(r_{e}\right)=V_{0}^{h}\left(r_{h}\right)= \begin{cases}0 & r<R \\ 0.08 \cdot E_{g}(M) & r \geq R\end{cases}$

where $R$ is the radius of the nanocrystal. We then apply the Hartre-Fock approximations at this point.

The Hartre-Fock equations are solved by employing the PMM (Bashkirov et al., 2011); subroutine for the calculation of the corresponding energies and wavefunctions (Poulopoulos et al., 2011) and thus the Hartree Fock potential. PMM solves the time-independent Schrödinger equation for an arbitrary interaction potential $v_{\mathrm{S}}(\vec{r})$ starting from a potential $v_{\mathrm{R}}(\vec{r})$ with well-known eigenvalues and eigenfunctions. The transition from potential $v_{\mathrm{R}}(\vec{r})$ to the potential $v_{\mathrm{S}}(\vec{r})$ by means of the time-dependent Schrödinger equations can then be derived as follows: Using the potential $v_{\mathrm{R}}(\vec{r})$ and $v_{\mathrm{S}}(\vec{r})$, the time-dependent Schrödinger equation (Goharshadi et al., 2009) can be formulated to be as follows;

$i \hbar \frac{\partial \Phi(\vec{r}, t)}{\partial t}=\left\{-\frac{\hbar^{2}}{2 m} \nabla^{2}+(1-\sigma(t)) v_{R}(\vec{r})+\sigma(t) v_{s}(\vec{r})\right\} \Phi(\vec{r}, t)$,

where $\sigma(\mathrm{t})$ has the following property;

$\sigma(t)=\left\{\begin{array}{ll}0, & t \leq t_{a} \\ 1, & t \geq t_{b}\end{array}\right.$.

For $t_{a} \leq t \leq t_{b}$ ( $t_{a}$ is the morphing starting moment, $t_{b}$ is the morphing ending moment (Gontard et al., 2011). The function $\sigma(t)$ should increase monotonically. After a large number of time steps (so that $t>t_{b}$ ), the energy eigenvalue $E_{S}$ for the potential $v_{\mathrm{S}}(\vec{r})$ can be given by

$$
E_{S}=\int d^{3} r \Phi_{S}^{*}(\vec{r})\left\{-\frac{\hbar^{2}}{2 m} \nabla^{2}+v_{S}(\vec{r})\right\} \Phi_{S}(\vec{r}),
$$

where $\Phi_{S}$ is the wave function of the system under consideration.

In the present calculations, the reference system for PMM is set to be the three-dimensional harmonic oscillator with the well known eigenfunctions (Liu et al., 2010)

$$
\Phi_{n l m}(r, \theta, \phi)=r^{l} e_{1}^{-\frac{m \omega}{2 \hbar} r^{2}} F_{1}\left(-n, l+3 / 2, \lambda r^{2}\right) Y_{l m}(\theta, \phi)
$$

where ${ }_{1} F_{1}\left(-n, l+3 / 2, \lambda r^{2}\right)$ is the hypergeometric function (Mahendia et al., 2011) making the interaction potential to become;

$v_{S}(\vec{r})=v_{H F}(\vec{r})+v_{C}(\vec{r})$,

where $v_{H F}(\vec{r})$ is the Hartree Fock potential for the electron (or hole), while $v_{C}(\vec{r})$ is the electron (or hole) confinement potential (Ning et al., 2010). When the procedure reaches a self-consistent solution, then the exciton energy is finally obtained by determining the sum of the corresponding electron and hole energies (Poulopoulos et al., 2011) as;

$E(X)=\tilde{E}_{e}+\tilde{E}_{h}$

and the effective band gap is given by; 
where $E_{g}$ is the bulk band gap energy.

\section{Experimental Procedures}

\section{Reagents and Chemicals}

Sodium sulfide hydrate, Triethanolamine (TEA), Tin (II) chloride dihydrate, polyvinyl alcohol (PVA) and absolute ethanol were obtained from Merck and used as-received without further purification.

\section{Required Apparatus and Instruments}

Philips X' Pert Pro with CuKa X-ray diffractometer, Carry 5 UV-Visible spectrophotometer, LEO 1430VP Scanning electron microscopy, Philips CN10, TEM performing were prepared and availed for characterization.

\section{Preparation of SnS nanocrystals Precusor solution}

$0.4 \mathrm{M}$ solution of tin chloride was prepared from $1.80 \mathrm{~g}$ of tin chloride powder is dissolved in $40 \mathrm{ml}$ TEA, then $0.62 \mathrm{~g}$ of sodium sulfide was dissolved in $40 \mathrm{ml}$ distilled water. These two solutions were mixed in a $200 \mathrm{ml}$ flask and maintained at a $\mathrm{pH}$ value of 10. The remaining procedure followed according to Ning et al., 2011 where the role of PVA is to stabilize the nanostructures preventing them from coagulation. The final products were collected for characterization using XRD, SEM, TEM, EDAX and UV-Visible spectroscopy.

\section{Proposed Reaction Mechanisms}

The formation mechanism for the formation of $\mathrm{SnS}$ nanocrystals was proposed as follows:

$$
\mathrm{SnCl}_{2} . \mathrm{H}_{2} \mathrm{O}_{(\mathrm{l})}+\mathrm{Na}_{2} \mathrm{~S} . \mathrm{xH}_{2} \mathrm{O}_{(\mathrm{l})} \longrightarrow \mathrm{SnS}_{(\mathrm{s})}+2 \mathrm{NaCl}_{(\mathrm{aq})}+\mathrm{xH}_{2} \mathrm{O}_{(\mathrm{l})}
$$

\section{Characterization of thin films}

Analysis of the coated films on an ordinary glass substrate from the SnS nanocrystals was carried out on a Philips $X^{\prime}$ Pert Pro X-ray diffractometer with $\mathrm{CuK} \alpha$ radiation while optical properties of were monitored using the Carry $5 \mathrm{UV}$ Visible spectrophotometer. Scanning electron microscopy (SEM) measurements and elemental analyses were performed using on a LEO 1430VP instrument operated at an accelerating voltage of $15 \mathrm{kV}$ while transmission electron microscopy (TEM) images of the sample were taken on a Philips CN10 at an accelerating voltage of $100 \mathrm{kV}$. First, the image was preprocessed by adjusting the contrast and brightness to minimise the speckle contrast of the background due to the carbon film used to support the sample. Secondly, the SnS nanoparticles were segmented using a semiautomatic procedure which combines interactive segmentation with adaptative thresholding, obtaining an mean particle diameter of $3.2 \mathrm{~nm}$ with a standard deviation of $1.9 \mathrm{~nm}$ as guided by Gontard et al. (2011), and Poulopoulos et al., (2011).

\section{RESULTS AND Discussion}

The SEM image showed that the product consisted of very small spherical SnS nanocrystallites aggregated in the form of polydispersive nanoclusters with sizes smaller than $100 \mathrm{~nm}$. From images it is clear that the sizes are in the order of very small nanoparticles. In order to further elucidate the morphology and the size of nanoparticles, Transmission Electron Microscopy (TEM) image was taken and a comparison of TEM and SEM images confirmed the formation of very small spherical SnS nanoparticles which most of them aggregated together in the form of polydispersive nanoclusters. The particle size distribution was also measured using the bright-field TEM images but the detection and clear manufestation of the measurement of the nanoparticles (segmentation) was difficult because thickness was belived to be changing locally, and diffraction from different crystal orientations introduce large contrast variations.

The quantitative analysis of the as prepared product was carried out using the EDAX technique and the resulting spectrum illustrated that the actual distribution of $S n$ and $S$ of the prepared sample. It was evident from the analysis that the product contains Sn and S materials with a same average atomic percentage ratio (1:1). The XRD pattern of as-prepared SnS nanocrystals showed several peaks corresponding to diffraction of orthorhombic SnS similar to Gontard et al., (2011). This clearly proved the polycrystalline nature of the as-prepared SnS which the appeared peaks are very consistent with the values in the standard card of SnS phase (JCPDS No. 39-0354).The broadness of the peaks obviously indicated nanocrystalline nature. The crystallite size of SnS nanoparticles was calculated using Debye-Scherrer formula (Vidal et al., (2012). 
$D=\frac{0.9 \lambda}{\beta \cos (\theta)}$

Here, $D$ is the coherent length, $\lambda$ the wave length of X-ray radiation, $\beta$ the full-width at half-maxima (FWHM) of the prominent peak and $\theta$ is the angle of diffraction. So the corresponding crystallite size of nanoparticles obtained smaller than $4 \mathrm{~nm}$ in the case of broadened peaks. Investigations proved that the absorption reduced rapidly with the increase of wavelength when it was analyzed using a plot of $(\alpha h v)^{2}$ versus photon energy $(h v)$ where the absorption coefficient $\alpha$, was calculated from the average absorption index $A$ as;

$\alpha=\frac{4 \pi A}{\lambda}$

The optical energy gap was evaluated by using the relation (Yue et al., (2009);

$a=\frac{A\left(h v-E_{g}\right)^{n}}{h v}$

where $A$ is an energy independent constant and $n$ characterizes the transition process. The fitted curve had a good straight line fit with higher energy range above the absorption edge, indicating a direct optical transition edge in which the direct energy gap of the sample was calculated as $1.74 \mathrm{eV}$ which was blue shifted in comparison the bulk band gap (Mahendia et al., 2011). Now in order to investigate the above system theoretically with PMM we By assuming that the matrix is PVA with $E_{g}(M)=4.98 \mathrm{eV}$ and by use the following material parameters as obtained from Vidal et al. 2012 for SnS: $m_{e}^{*}=0.5 m_{0} m_{h}^{*}=0.109 m_{0}$, where $m_{0}$ is the electron mass, $\varepsilon_{0}=32$ and Mahendia et al., (2011) as well as from Yue et al. (2009) with values of $\varepsilon_{\infty}=16$ and $\hbar \omega_{L O}=71 \mathrm{meV}$ and $E_{g}($ bulk $)=1.296 \mathrm{eV}$, modeling was carried out using $\mathrm{Eq}(2.10)$ to $\mathrm{Eq}(2.15)$ and the results were fairly in good agreement with the size which is obtained from the Debye-Scherrer formula and TEM image analysis.

\section{CONCLUSION}

In conclusion, very simple and not expensive procedure was used to synthesize SnS nanocrystals at normal laboratory conditions. The as-synthesized $\mathrm{SnS}$ nanoparticles appeared to have strongly blue shifted direct band gap energy with a value $1.74 \mathrm{eV}$. Theoretical curve based on the potential morphing method of the exciton energy versus the particle size obtained through PMM method in the Hartree Fock approximation, showed a fairly blue shift that was attributed to the effect of the quantum confinement. In this stuudy, the PMM based results exhibited a fairly good agreement with the experimental data hence providing a better insight on the quantum confinement effects in SnS nanoscaled systems.

\section{ACKNOWLEDGEMENTS}

The authors acknowledge the support by Technical University of Mombasa - (TUM) and Masai Mara University (MMU) in carrying out this work. This research has been also co-financed by the authors and the department of Mathematics and Physics of the Technical University of Mombasa.

\section{REFERENCES}

Azizian-Kalandaragh Y. \& Khodayari A., 2010. Aqueous synthesis and characterization of nearly monodispersed ZnS nanocrystals, Physica Status Solidi a, 207, 9, 2144-2148.

Azizian-Kalandaragh Y. \& Khodayari A., 2010. Ultrasound-assisted preparation of CdSe nanocrystals in the presence of Polyvinyl alcohol as a capping agent. Mater. Sci. Semicond. Process. 13, 225-230.

Azizian-Kalandaragh Y., Khodayari A., \& Behboudnia M., 2009. Ultrasound-assisted synthesis of ZnO semiconductor nanostructures. Mater. Sci. Semicond. Process. 12, 142-145.

Bashkirov S.A., Gremenok V.F., \& Ivanov V.A., 2011. Physical properties of SnS thin films fabricated by hot wall deposition. Fizika i Tekhnika Poluprovodnikov 45, 765-769.

Goharshadi E.K., Ding Y., Jorabchi M.N. \& Nancarrow P., 2009. Ultrasound-assisted green synthesis of nanocrystalline ZnO in the ionic liquid [hmim] [NTf 2 ]. Ultraso. Sonochem. 16, 120-123. 
Gontard L.C., Ozkaya D. \& Dunin-Borkowski R., 2011. A simple algorithm for measuring particle size distributions on an uneven background from TEM images. Ultramicroscopy 111, 101-106.

Liu H., Liu Y., Wang Z., \& He P., 2010. Facile synthesis of monodisperse, size-tunable SnS nanoparticles potentially for solar cell energy conversion. Nanotechnology 21, 105707.

Mahendia S., Tomar A.K., Chahal R.P., Goyal P. \& Kumar S., 2011. Optical and structural properties of poly(vinyl alcohol) films embedded with citrate-stabilized gold nanoparticles. J. Phys. D 44, 205105.

Ning J., Men K., Xiao G., Wang L., Dai Q., Zou B., Liu B. \& Zou G., 2010. Facile synthesis of IV-VI SnS nanocrystals with shape and size control: Nanoparticles, nanoflowers and amorphous nanosheets. Nanoscale 2, 1699-1703.

Poulopoulos P., Baskoutas S., Pappas S.D., Garoufalis C.S., Droulias S.A., Zamani A. \& V. Kapaklis, 2011. Intense Quantum Confinement Effects in $\mathrm{Cu}_{2} \mathrm{O}$ Thin Films. J. Phys. Chem. C 115, 14839-14843.

Vidal J., Lany S., d'Avezac M., Zunger A., Zakutayev A., Francis J. \& Tate J., 2012. Band-structure, optical properties, and defect physics of the photovoltaic semiconductor SnS. Appl. Phys. Lett. 100, 032104-032107.

Yue G.H., Peng D.L., Yan P.X., Wang L.S., Wang W. \& Luo X.H., 2009. Structure and optical properties of SnS thin film prepared by pulse electrodeposition. J. Alloy \& Compounds 468, 254-257. 